\title{
Surface vs diffusion in TDS of hydrogen
}

\author{
Ekaterina L. Alekseeva ${ }^{1,2}$, Alexander K. Belyaev ${ }^{2,1}$, Anatoly M. Polyanskiy ${ }^{2}$, Vladimir A. Polyanskiy ${ }^{2,{ }^{*}}$, Evgeniy A. \\ Varshavchik $^{2,1}$ and Yuri A. Yakovlev, ${ }^{2}$ \\ ${ }^{1}$ Peter the Great St. Petersburg Polytechnic University (SPbPU), 195251 Polytechnicheskaya, 29, St. Petersburg, Russia \\ ${ }^{2}$ Institute for Problems in Mechanical Engineering of Russian Academy of Sciences, 199178 V.O., Bolshoy pr., 61, St. Petersburg, \\ Russia
}

\begin{abstract}
The paper addresses the numerical simulation of conditions in which the measurement of thermal desorption spectra of hydrogen (TDS) is carried out. Plane steel samples of $10 \mathrm{~mm}$ thickness were used as the specimens for simulation. The skin effect which is observed with standard hydrogen charging of samples was accounted for the initial conditions. The standard diffusion of hydrogen was simulated according to Fick's law. Solution of the Fick's equation is obtained by finite element methods using the developed code. The resulting solutions show that standard hydrogen charging can lead to the appearance of an additional TDS peak, even without taking into account the traps. New interpretation of the TDS method for hydrogen dissolved in a solid was suggested.
\end{abstract}

\section{Introduction}

Hydrogen which is dissolved in metals is the most dangerous component. It begins to induce destruction processes in some metals starting with the mass concentrations of $0.5 \mathrm{ppm}$.

Hydrogen can be absorbed by the metal during its production. However the main amount of hydrogen frequently leaks from the environment due to diffusion.

The rate of hydrogen absorption by a metal depends on its chemical composition, structure, mechanical loading conditions, temperature, and chemical composition of the environment. The metals having minimum sensitivity to the environmental hydrogen are used in oil and gas projects. The standard sensitivity testing consists in saturating metallic samples with hydrogen and subsequent investigation of changes in the microstructure and mechanical properties of the samples. Typically, the standard time for saturation or charging of samples with hydrogen is less than 100 hours.

Naturally, the development of new materials for the oil and gas industry is accompanied by the same testing. To analyze the results of hydrogen saturation, the method of thermo-desorption spectra (TDS) cf. [1,2] is additionally applied. Almost all modern studies of hydrogen interaction with metals are accompanied by measurement of TDS. All references to these studies are impossible to list in one article.

An important feature of this method is the ability to determine the binding energies of hydrogen in the metal. The model of the hydrogen extraction from samples under the TDS process was reported in [3]. Currently, TDS is the main method for determining the binding energies of hydrogen in metals. However the diffusion of hydrogen in samples was ignored there. The process of extracting hydrogen from a metal sample during its slow heating at a constant rate is modeled as a first-order chemical reaction. The discrepancy between the diffusion nature of hydrogen extraction and its modelling results in different values of binding energies for samples of different shapes and dimensions.

We investigated these inconsistencies in detail, cf. [47]. They arise not only as a result of ignoring diffusion, but are based on two other important reasons: the Oriani's trap-diffusion model [8] and the notions of uniform distribution of internal hydrogen after artificially charging metallic samples according to standardized methods.

In fact, the Oriani model assumes the presence of a multicomponent mixture inside a metal sample, desorbing from the hydrogen traps of different nature. The decomposition of this mixture by heating the samples leads to the hydrogen desorption with the rate depending on the temperature. In this case, a single diffusion channel is taken into account, see [8].

An important fact indicated by users of the TDS method is that the spectra have several peaks. The general opinion is that the multi-peaks can be explained only by the presence of hydrogen traps of different nature, uniformly distributed throughout the volume of the metal and having different binding energies with hydrogen. A lot of articles are devoted to the justification of this approach, cf. [9-15]. All the authors point out that each peak of TDS can be associated with the binding energy of a particular trap. In some cases there is a single peak. All the same, this peak is considered as a superposition of hydrogen fluxes from traps with different binding energies and is laid out using an approximation method into smaller ones. In this case,

* Corresponding author: vapol@mail.ru 
the authors proceed from a priori considerations about the assumption of the type of trap in the alloy, cf.[16,17].

Some experimental data cast doubt on the adequacy of this approach cf. $[5,7]$. With this in view, we propose a new approach to the problem considered.

\section{Hydrogen charging effect}

Using the TDS method is problematic without charging samples with hydrogen. Most metals, except for alloys of magnesium, zirconium and titanium, have very small natural concentrations of hydrogen (the so called metallurgical hydrogen). For example, these concentrations are less than $1 \mathrm{ppm}$ for steels which are not affected by a corrosive or stress-corrosive process and less than $0.2 \mathrm{ppm}$ for aluminum alloys.

Recently published article [18] declared the possibility of mass-spectrometric measurement of TDS for such concentrations. But the authors themselves found out that water, which is always adsorbed from the atmosphere on the surface of metal samples, introduces a very significant error in the spectrum.

Practically nobody proves the uniformity of the concentration distribution at standardized charging with hydrogen. It is believed that tens of hours is enough for uniform charging. In many papers, the concentration of hydrogen is not measured at all. The charging time is taken as a parameter corresponding to the concentration of charged hydrogen, and for cathodic charging the charge current and the saturation time are taken as such parameters.

However, there are several works in which the distribution of hydrogen concentrations after standard charging was measured, see [19-23]. This work covers all standardized methods of charging samples: in gaseous hydrogen, in saline solution and cathode charging.

Fig. 1 shows the concentration of hydrogen distribution diagram which was averaged over the layer thickness of $0.05 \mathrm{~mm}$. The scale of hydrogen concentration is logarithmic. This experimental data we obtained on cylindrical samples diameter $8 \mathrm{~mm}$ of bridge steel after 96 hours of standard hydrogen charging in a neutral salt solution, according to according to NACE TM0284-2016.

There are several more calculated results that confirm the experimental ones [24-26]. All the experiments and simulation data unequivocally prove that without strong heating an uneven concentration appears under the standard conditions of the hydrogen charging of samples. Moreover, the values of concentration in a thin surface layer with a thickness of $50-100$ microns about a hundred times greater than the average concentration of hydrogen inside the sample. The equalization of concentrations occurs after the hydrogen saturation for 500-600 hours, see [19].

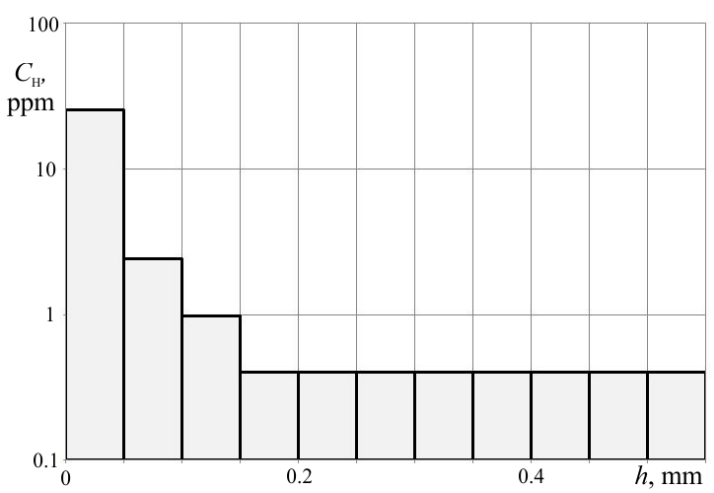

Fig. 1. Hydrogen concentration averaged over the layer thickness of $0.05 \mathrm{~mm}$ vs the distance from the surface of the cylindrical sample of bridge steel after standard hydrogen charging according to NACE TM0284-2016 in a neutral salt solution.

Thus, it is possible to represent the distribution of the concentration of hydrogen as a function shown in the graph Fig.2.

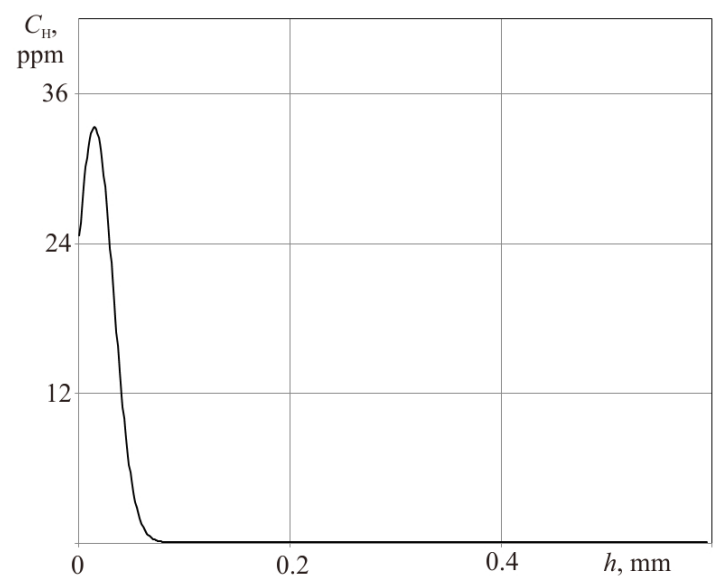

Fig. 2. The most likely hydrogen concentration vs distance $h$ from the surface of the cylindrical sample that corresponds to the test results of Fig. 1.

There is a number of indirect evidences of exactly this type of distribution of hydrogen concentrations.

For example, it is known that a slight heating to temperature of $30-50{ }^{\circ} \mathrm{C}$ leads to strong degassing of the samples results. This is due to the great gradients of hydrogen concentration near the surface of the sample which leads to large flows of hydrogen even in the cold state. The time interval between charging with hydrogen before the measurement of TDS usually takes from several hours to several days. The concentration of hydrogen stabilizes with time however any increase in the diffusion coefficient leads to a new emission of hydrogen from the sample. In the analysis of TDS, this hydrogen is associated with dislocation traps, although it can be a simple consequence of the non-uniform saturation with hydrogen.

This distribution also explains the fact that long outside exposure of samples in many cases leads to the almost complete disappearance of the effects associated with the hydrogen charging. There exists even the term "reversible hydrogen embrittlement". Indeed, large concentration gradients and a small depth of the 
hydrogen penetration results in a complete disappearance of hydrogen from the sample even at the room temperature.

\section{Simulation of the charging effect in TDS}

The TDS test requires the charged samples be inserted into the TDS chamber where they heat up at a constant rate. The hydrogen flux desorbed from samples is measured by means of various techniques. The dependence of the hydrogen flux on the sample temperature is referred to as TDS. The main elements of the spectrum are hydrogen flux peaks.

The analysis of TDS data ignores hydrogen diffusion during the TDS measurement. This approach requires that experimental details minimize the influence of hydrogen diffusion. There is no consensus on this subject, despite fifty years of experience in using TDS. For example, Lee and Lee [27] demonstrated the impact of hydrogen diffusion. Quite the contrary, Ono and Meshii [28] believe that the influence of diffusion could be ignored when the sample was sufficiently thin and was subjected to the adequate surface treatment. In opposite, Turnbull et al. [29] concluded that diffusion can only be neglected for limiting circumstances, such as for low alloy steels at low hydrogen contents.

The activation energy was determined by performing TDS tests using four different heating rates $\varphi$, namely $0.1,0.2,0.3$, and $0.4 \mathrm{~K} / \mathrm{s}$. Equation (1) is a simplification of the original formula by Kissinger [3]:

$$
\ln \left(\varphi / T_{\mathrm{p}}^{2}\right)=\text { Const- } E_{\mathrm{p}} /\left(\mathrm{R} T_{\mathrm{p}}\right)
$$

where $T_{\mathrm{p}}$ is the peak temperature $(\mathrm{K}), \mathrm{R}$ is the molar gas constant $(8.31447 \mathrm{~J} /(\mathrm{mol} \cdot \mathrm{K}))$ and $E_{\mathrm{p}}$ is the activation energy corresponding to the peak ( $E_{\mathrm{p}}$ may be determined graphically).

We use the experimental data obtained and model the pure diffusion of hydrogen according to the Fick law (2), and Arrhenius low (3) without any traps.

$$
\begin{gathered}
\partial \mathrm{C} / \partial t=D(T) \Delta C \\
D(T)=D_{0} \exp \left(-U_{\mathrm{a}} / \mathrm{R} T\right) \\
T=T_{0}+\varphi t
\end{gathered}
$$

where $T$ is the temperature (K), $t$ is the time (s), $C$ is the hydrogen concentration (ppm), $U_{\mathrm{a}}$ is the diffusion activation energy $(\mathrm{J} / \mathrm{mol})$ and $D_{0}$ is the diffusion constant $\left(\mathrm{m}^{2} / \mathrm{s}\right)$.

For modeling we took a flat steel sample with a thickness of $10 \mathrm{~mm}$. We will consider the onedimensional problem of pure diffusion of hydrogen with an activation energy $U_{\mathrm{a}}=35 \mathrm{~kJ} / \mathrm{mol}$, see (3) and the heating rate $\varphi=0.015 \mathrm{~K} / \mathrm{s}$. The initial distribution of the hydrogen concentration is shown in Fig.3.

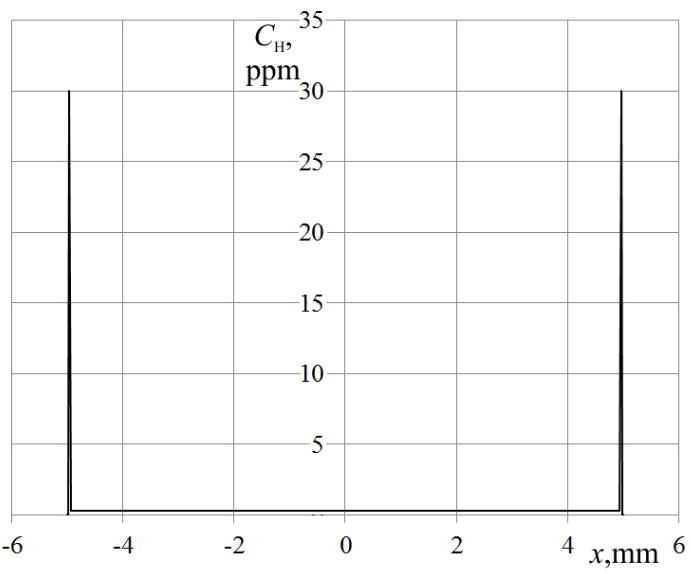

Fig. 3. Hydrogen concentration in the simulated sample

The solution of the differential equation (2) is given by the finite element method. The standard difference scheme for the finite element approximation of equation (2) is an implicit one, so we will solve it by the method of tridiagonal sweep.

For the numerical solution of this difference scheme, $\mathrm{C}++$ code was developed. The simulation results are presented in Figs.4-6. The calculated distributions of the hydrogen concentration in the sample at different temperatures of TDS are displayed in Fig.4.

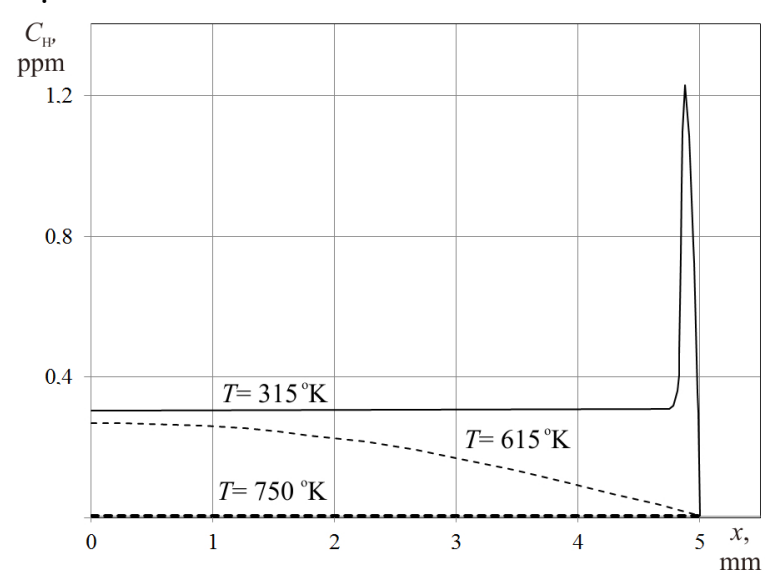

Fig. 4. Hydrogen concentration vs coordinate $x$. A half of the symmetrical distribution is given.

Figs .5 and 6 show TDS for temperatures $315 \mathrm{~K}$ and $750 \mathrm{~K}$.

The simulation results show that the peaks in the TDS may have nothing in common with the hydrogen traps having different binding energies. 


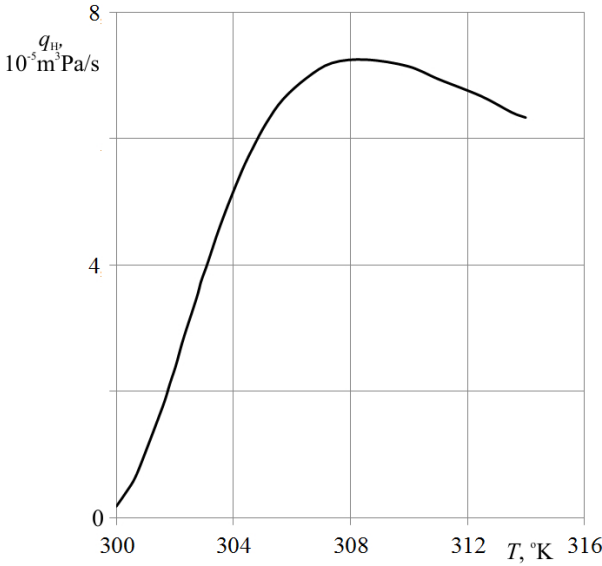

Fig. 5. Simulated TDS for the temperature range $300-315 \mathrm{~K}$.

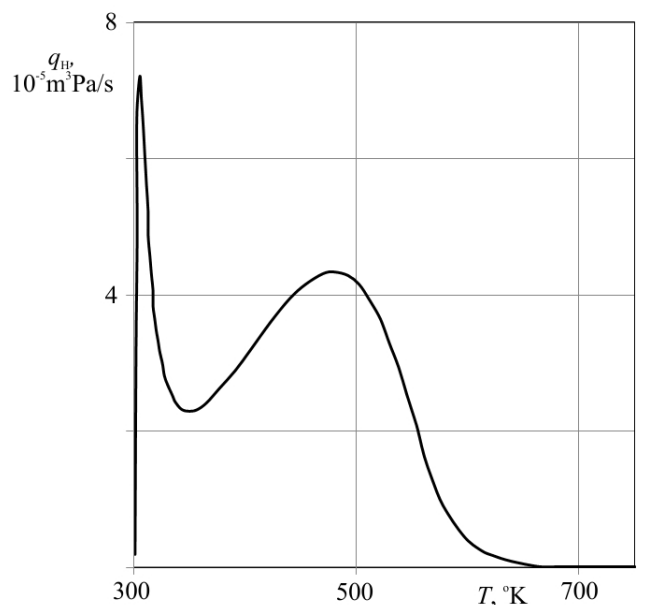

Fig. 6. Simulated TDS for the temperature range 300-750 K.

The conventional diffusion (according to Fick's law) of hydrogen at the non-uniform distribution can give the same effect as hydrogen traps with different binding energy. It is important that with standard charging with hydrogen, a non-uniform concentration occurs. There is a doubt about the adequacy of all the results obtained by using TDS.

There is a doubt in the adequacy of all the results obtained using TDS without checking the uniformity of charging with hydrogen. Moreover, a number of studies, cf. [26-28], show that the lack of diffusion in the Kissinger [3] model, see [3] can lead to significant errors in the determination of the binding energy of hydrogen traps based on the Oriani model cf. [8].

On the other hand, the experimentally observed surface effect of charging samples with hydrogen corresponds well to the TDS method, which is essentially superficial for the solid samples. Any diffusion of hydrogen introduces errors in the magnitude of its binding energies.

Thus, most of the available results adequately reflect the peculiarities of surface sorption of hydrogen when charging samples.

\section{Conclusions}

We conducted a finite-element simulation of the diffusion flux of hydrogen from a metal steel sample heated at a constant rate.

The majority of the available results adequately reflect the peculiarities of surface sorption of hydrogen when charging samples. Such a distribution of hydrogen concentrations was chosen for modeling, which appeared in the process of standard charging of samples using the different methods.

We have obtained that the TDS peaks may not be related to the binding energy of hydrogen in different types of traps. There may be peaks caused by the nonuniform distribution of hydrogen.

The research is carried out under the financial support by Russian Science Foundation, grant 18-19-00160.

\section{References}

1. R. P. Aveling, U.S. Patent No. 3,068,402. (1962).

2. F. J. Castro, G. Meyer, Journal of alloys and Compounds 330 59-63 (2002).

3. H. E. Kissinger, Analytical Chemistry 29111702 1706 (1957)

4. A. M. Polyanskiy, V. A. Polyanskiy, Y. A Yakovlev, International Journal of Hydrogen Energy 3930 17381-17390 (2014)

5. D. Y. Andronov, D. G. Arseniev, A.. M. Polyanskiy, et al. International Journal of Hydrogen Energy 421 699-710 (2017)

6. D. G., Arseniev, A.K. Belyaev, V.A. Polyanskiy, et al. Dynamical Processes in Generalized Continua and Structures (pp. 37-61 Springer, Cham 2019).

7. A. K. Belyaev, A. M. Polyanskiy, V. A. Polyanskiy, et al. International journal of hydrogen energy $41208627-8634$ (2016)

8. R. A. Oriani Acta metallurgica 181 147-157 (1970)

9. E. J. Song, D.W. Suh, H. K. D. H. Bhadeshia Comp. Mat. Sci. 79 36-44 (2013)

10. R. Kirchheim Metall. Trans. A 47 672-696 (2016)

11. P. D. Escobar, T. Depover, L. Duprez, et al. Acta Mater. 60 2593-2605 (2012)

12. F. J. Castroa , G.1 Meyerb Journal of Alloys and Compounds 330-332 59-63 (2002)

13. N. I. Rodchenkova, Y. V. Zaika Journal of Physics: Conference Series 661012026 (2015)

14. Y. V. Zaika, N. I. Rodchenkova Appl. Math. Modelling 33 3776-3791 (2009)

15. T. Schablitzki, J. Rogal, R. Drautz Phil. Trans. R. Soc. A 37520160404 (2017)

16. T. Yokota, T. Shiraga ISIJ International 434534 $538(2003)$

17. E. Steppan, P. Mantzke, B. R. Steffens, et al. Welding in the World 614 637-648(2107) 
18. C.V. Tapia-Bastidas, A. Atrens, E. MacA. Gray International Journal of Hydrogen Energy 4315 7600-7617 (2018)

19. A. Martinsson, R. Sandström, Journal of Materials Science 4719 6768-6776 (2012)

20. R. Wu, J. Ahlström, H. Magnusson, K. Frisk, A. Martinsson, Charging, degassing and distribution of hydrogen in cast iron, Swerea KIMAB (2015)

21. A.-M. Brass , J. Che Corrosion Science 483222 3242 (2006)

22. M. Enomoto, L. Cheng, H. Mizuno, et al. Metallurgical and Materials Transactions E 14331 340 (2014)

23. E. L. Alekseeva, A. K. Belyaev, A. S. Zegzhda, et al. Diagnostics, Resource and Mechanics of materials and structures 3 43-56 (2018)

24. T. Omura, J. Nakamura, H. Hirata, et al. ISIJ International 563 405-412(2016)

25. Y. Yagodzinskyy, O. Todoshchenko, S. Papula, et al. Steel research international 821 20-25 (2011)

26. U. Hadam, T. Zakroczymski International Journal of Hydrogen Energy 345 2449-2459 (2009)

27. S.M. Lee, J.Y. Lee, Metallurgical Transactions A 17 2 181-187 (1996)

28. K. Ono, M. Meshii, Acta metallurgica et materialia 406 1357-1364 (1992)

29. A. Turnbull, R.B. Hutchings, D.H. Ferris, Materials Science and Engineering A 2382 317-328 (1997) 